\title{
Interview with Stephen B Baylin
}

Epigenomics

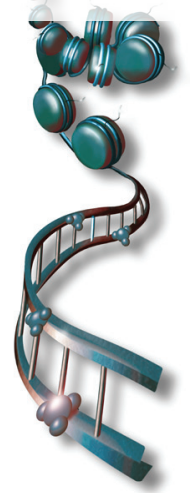

Stephen B Baylin is a codirector of the Cancer Biology Program at The Sidney Kimmel Comprehensive Cancer Center at Johns Hopkins and the Virginia and DK Ludwig Professor of Oncology and Medicine. Baylin attended Duke University, where he earned his medical degree and completed his internship and first year residency in internal medicine. He then worked for 2 years at the National Heart and Lung Institute of the NIH. In 1971, he joined the departments of oncology and medicine at Johns Hopkins University School of Medicine. His research interests include cellular biology and genetics of cancer, specifically epigenetics or genetic modifications other than those in DNA that can affect cell behavior, and silencing of tumor suppressor genes and tumor progression. His research has looked at the mechanisms through which variations in tumor cells derive, and cell differentiation in cancers such as medullary thyroid carcinoma and small-cell lung carcinoma. He has served on the American Association for Cancer Research Board of Directors, and is an associate editor of Cancer Research. He has also presented frequently at AACR conferences and chaired the special conference on 'DNA Methylation, Imprinting and the Epigenetics of Cancer'. He has authored or coauthored more than 370 publications.

Q Dr Baylin, you are the codirector of the Cancer Biology Program of The Sidney Kimmel Comprehensive Cancer Center at Johns Hopkins \& the Virginia \& DK Ludwig Professor of Oncology \& Medicine. Can you tell our readers a little about how you became involved in this field of work? What have been your greatest successes to date?

I became involved somewhat through chance while studying basic aspects, during the 1970s and 1980s, of signal transduction responsible for hormonal production in various human cancer types. We discovered, in the mid 1980s that the promoter of the small polypeptide hormone gene, calcitonin, contained abnormal amounts of DNA methylation in cancer cells as compared with normal tissues that lacked this modification in the promoter. This started us off on a hunt for the importance of such a change in cancer. The result has been a body of work over the subsequent years showing that promoter hypermethylation of normally unmethylated $\mathrm{CpG}$ island regions is a fundamental characteristic of human cancers of virtually all types now studied by ourselves and others. This can occur in bonafide tumor suppressor genes in association with abnormal transcriptional repression to constitute an alternative to mutations for their functional inactivation. We have also had success in building data to delineate causes of this abnormality and are most excited by the role of oxidative damage and the protein complexes involved, that may help evolve this change during tumor initiation and progression. Finally, we have helped developed strategies to search the genome agnostically for genes affected by the above change and mouse knockout strategies proving that the change, even in genes without a history of mutations in cancer, can constitute as tumor suppressor function. Along with these approaches, we have outlined how the

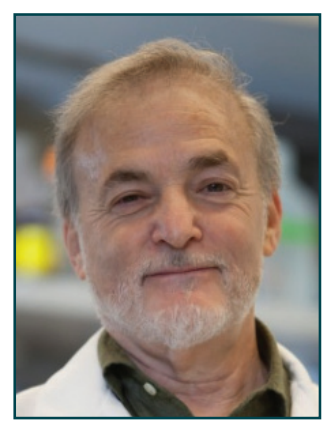

Stephen B Baylin The Sidney Kimmel Comprehensive Cancer Center at Johns Hopkins, Baltimore, MD 21287, USA sbaylin@jhmi.edu
Future 
change affects multiple points in key signaling pathways to produce abnormalities in their function in cancer.

What most excites you about the field of epigenetics/epigenomics at the moment?

I am most excited about the translational implications for cancer that are the consequences of the seamless interactions between today's most fundamental and clinical research efforts in epigenetics. This creates an understanding that we can especially build on for new approaches for cancer therapies. I build on this in the section just below.

Q Can you tell our readers a little about some of the recent research that has been going on this field?

As alluded to just above, our growing fundamental understanding of how proteins and protein complexes regulate normal and cancer epigenomes is allowing us to take older drugs, such as inhibitors of DNA methylation and histone deacetylase inhibitiors, plus newer drugs which target various steps in epigenome regulation, and devise new strategies for cancer therapy. This includes combinatorial strategies for such of the epigenetic drugs that might be employed with other cancer therapies. One particular example of all of this is the current clinical trial and laboratory work our group is now heavily engaged in pursuing the hypothesis that epigenetic therapy can sensitize patients with lung, ovarian, colon and other cancers to immune checkpoint therapy. This latter therapy is arguably the most exciting advance over the last few years for potentially achieving durable management of advanced human cancers. Despite rapid advances, immune checkpoint therapy given alone, for melanoma and a subgroup of colon cancers, does not provide response fore most patients. What we are learning is that combining this approach with epigenetic therapy may constitute a robust means for achieving such improved therapeutic responses. We have derived a molecular signature indicating that an older epigenetic drug, the DNA methyltransferase inhibitor, 5-aza-cytidine can prime human cancer cells to augment immune attraction by a complex upregulation of an antigen processing, inflammatory mediating and interferon driving immune attraction response. The core pathway for this is upregulation of a dsRNA sensing pathway for viral defense. Not only is this the case, but basal expression of delimited viral defense gene panel looks to provide a robust prediction for response to checkpoint inhibitors in an actual clinical trial. Tumors are generally high or low for this signature - for example, a high signature encompasses a previously defined TCGA category of immunoreactive ovarian cancers. So, our hypothesis is that epigenetic therapy which activates this signal in tumors that are low, might augment responses to immune checkpoint therapy.

Q What are the major implications that this research has on cancer diagnosis \& management? The answer just above mentioned highlights this implication. Also, epigenetic signatures, such as abnormal sites of DNA methylation are building toward clinical use for biomarker strategies for early cancer detection and for predicting therapy responses.

Q What are the main hurdles/caveats that the field faces with regard to translating basic research into changes in clinical practice?

Translation of promising laboratory findings predictive for therapy efficacy often falters at the level of ultimate proof - that is, testing in a clinical trial. We need to learn to conduct the most predictive early trials that allow progress in extending the hypotheses. Often a trial looks like a failure, not because the proposed paradigm is wrong, but the precise way to perform the therapy (how to dose and sequence the drugs involved, for example) has not been properly performed in the first approach. We need to be more clever in how the first trials are performed.

Q How best can/should these hurdles be overcome, in your opinion?

As above explained, we must learn to marry the fundamental and preclinical research with initial trial design.

\section{Q Do you have any exciting projects in the} pipeline that you would like to share with our readers?

I think the above mentioned research to combine epigenetic therapy with immune checkpoint therapy for cancer is terribly exciting and building. Two papers now in press, in Cell, one from De Carvalho and colleagues and one from our group, highlight the possibilities of this entire endeavor - these involve upregulation of the viral defense pathway alluded to above, including a driving role for aza-cytidine-induced upregulation of endogenous retroviral transcripts. This brings in the oft-forgotten role of the epigenome in regulating transcription of noncoding regions in our genome and how to translate this knowledge for cancer biomarker and therapy strategies.

\section{Q What direction do you see the field of epigenomics taking in general over the next 5-10 years?}

Our understanding of the epigenome and its normal and abnormal regulation is going to increase at the pres- 
ent rapid rate. The fundamental knowledge emerging is going to provide ever increasing ramifications for translational science in areas such as cancer. New targeting strategies, as mentioned above, are going to allow us to build on already exciting indications that epigenetic therapy could provide a tremendous component for cancer therapy - and I predict therapies for other diseases as well such as neurodegenerative disorders, diabetes, and so on.

\section{Disclaimer}

The opinions expressed in this interview are those of the interviewee and do not necessarily reflect the views of Future Medicine Ltd.

\section{Financial \& competing interests disclosure}

SB Baylin consults for MDx Health in agreement with the Johns Hopkins School of Medicine. MDx Health licences, from the School of Medicine, the technology of methylation specific PCR for detection of DNA methylation abnormalities and is entitled, as well as the author, to royalties from sales involved with this technology. SB Baylin has no other relevant affiliations or financial involvement with any organization or entity with a financial interest in or financial conflict with the subject matter or materials discussed in the manuscript apart from those disclosed.

No writing assistance was utilized in the production of this manuscript. 